\author{
KRZYSZTOF KOTUŁA \\ Uniwersytet Marii Curie-Skłodowskiej w Lublinie \\ christophe.kotula@gmail.com \\ ORCID: 0000-0002-9113-7779
}

\title{
Le doublage comme un moyen de travailler sur la prononciation française : théorie et pratique
}

\author{
Video dubbing as a method for French \\ pronunciation training: theory and practice
}

\begin{abstract}
Pronunciation is an often-neglected aspect of language instruction. Teachers usually place the blame on lack of time and resources, as well as curricular restrictions. However, in recent years, we have witnessed a proliferation of various tools that can be used to render work on different aspects of pronunciation much more interesting. In the present paper, we will focus on one particular technique: video dubbing. By examining the answers provided by the participants of a dubbing project conducted by the author of the present study, we will try to reflect on the potential advantages of such activities, and determine whether they are considered stimulating and effective by language learners.
\end{abstract}

KEYWORDS: computer-assisted language learning, pronunciation training, video dubbing.

MOTS-CLÉS : apprentissage des langues assisté par ordinateur, enseignement de la pronunciation, doublage

\section{INTRODUCTION}

Personne ne conteste que la maîtrise de la prononciation est importante dans l'apprentissage efficace des langues étrangères. Gajewska souligne avec raison que l'incapacité à différencier les phonèmes, à lire et à prononcer correctement les mots inconnus affecte lourdement tout le processus d'apprentissage d'une langue étrangère ; elle remarque également qu'un élève chez qui persiste une prononciation incorrecte est un élève à qui on a fait du tort (Gajewska 2011). 
Pourtant, on constate souvent que la phonétique est marginalisée dans l'enseignement, souvent à cause de la persistance de certaines croyances entièrement fausses, telles que la conviction que passé un certain âge, il est absolument impossible de maîtriser la prononciation correcte d'une langue étrangère ou que " la simple exposition à la langue-cible, le "bain linguistique", serait suffisante pour assurer à l'apprenant une bonne prononciation » (Hermeline 2001 : 27). La situation est encore compliquée par les contraintes qui pèsent sur le contexte institutionnel. Comme le souligne Baran-Łucarz (2006), les enseignants expliquent la tendance à négliger la prononciation par des facteurs externes, tels que l'absence de ressources pour enseigner cet aspect, le manque d'intérêt de la part des élèves, le caractère ennuyeux des exercices, la surcharge des programmes scolaires et les contraintes éducationnelles parmi lesquelles la prononciation joue un rôle mineur.

De nombreux spécialistes déplorent que le travail sur la prononciation correcte soit relégué au second plan ; ils soulignent que c'est un élément clé dans l'apprentissage d'une langue " et qu'il pourrait en être l'un des plus ludiques" (Parizet 2008: 114). En effet, plusieurs exercices possèdent un potentiel incontestable pour séduire les apprenants : comme le souligne Parizet, « on peut [leur proposer] de lire à voix haute des vire-langues ou de petits poèmes, existants ou créés par eux-mêmes, ou encore de chanter des chansons comportant le ou les phonèmes retenus » (Parizet $2008: 121)$. Il est évidemment nécessaire de rompre la routine et de proposer aux apprenants des activités créatrices qui ne se limitent pas à l'écoute et à la reproduction des sons (ou séquences de sons) entendus.

Dans notre article, nous allons nous concentrer sur les activités de doublage, qui permettent aux apprenants de travailler d'une manière innovante sur la prononciation de la langue française tout en mobilisant le potentiel des nouvelles technologies. Dans un premier temps, nous allons nous interroger sur la place des TICE dans l'apprentissage de la phonétique de la langue française en présentant quelques outils essentiels. Dans un deuxième temps, nous allons passer en revue les recherches se focalisant spécifiquement sur le doublage en classe de langues. Finalement, nous allons décrire le projet de doublage que nous avons réalisé dans le cadre du cours de phonétique française au cours de l'année universitaire 2014/2015. Nous allons présenter les opinions des participants et nous demander quels sont les facteurs qui ont pu les influencer. À la fin, nous allons nous interroger sur la manière optimale d'implémenter les projets de doublage dans l'enseignement de la prononciation et tenter de formuler quelques indications pratiques qui pourraient servir aux enseignants de langue française qui désireraient incorporer ce genre d'activités dans leur pratique de classe. 


\section{LA PLACE DES TICE DANS L'ENSEIGNEMENT DE LA PRONONCIATION}

Dans les récentes années, les méthodes de l'enseignement de la prononciation ont considérablement évolué. Le premier changement important s'est opéré suite à l'apparition d'outils qui rendent possible l'enregistrement et une analyse minutieuse de la prononciation des apprenants. Le deuxième changement a plutôt consisté en une réorientation des priorités: « moving beyond an emphasis on the accurate production of individual speech sounds to concentrating more on the broader, communicative aspects of connected speech » (Richards, Renandya 2002 : 175). Ces deux aspects sont naturellement étroitement liés, car le développement technologique permet d'élaborer des méthodes de plus en plus performantes permettant de travailler sur les aspects complexes de la prononciation.

Le nombre d'outils permettant de rendre le travail sur la prononciation plus intéressant et efficace est important et en croissance constante. Le réseau Internet constitue un vaste répertoire d'outils librement disponibles dans lequel un enseignant désireux de rompre avec la monotonie des leçons peut puiser à volonté : enregistrements vidéo expliquant les principes de la prononciation et démontrant le travail correct de l'appareil articulatoire, exercices interactifs, jeux... Le nombre de ressources, créées le plus souvent par des passionnés de la langue française et mises gratuitement à la disposition des internautes, est quasi-illimité. Leur rôle ne se limite pas à enrichir les cours de langue : ces ressources peuvent également contribuer à l'autonomisation de l'apprenant en lui permettant de travailler sur certains aspects de la prononciation dans le milieu extrascolaire. En effet, de nombreux exercices interactifs incorporent un mécanisme de rétroaction immédiate permettant à l'élève de suivre ses progrès en temps réel ainsi que d'identifier les problèmes persistants.

Nombreux sont également les outils qui n'ont pas été créés pour servir un objectif éducatif, mais qui peuvent malgré tout être utilisés dans le contexte pédagogique. Parmi ceux-ci, il faut mentionner en premier lieu les logiciels de synthèse vocale, permettant de transformer le texte écrit en texte parlé. Le développement très rapide de cette technologie dans les dernières années rend possible une imitation convaincante du locuteur humain (Kotuła 2014). C'est une aide inestimable permettant aux apprenants de faire des progrès sans avoir besoin de connaître l'alphabet phonétique. Nous pouvons ranger dans la même catégorie les logiciels qui permettent de créer une représentation graphique d'un enregistrement sonore (Rojczyk 2011; Olson 2014). Ces représentations (oscillogrammes, courbes d'amplitude et mélodiques, sonagrammes, etc.) permettent de visualiser les aspects choisis de la 
prononciation de l'apprenant. Le problème principal réside dans la difficulté d'interprétation des données fournies par ces logiciels, car l'interprétation des courbes sonores exige un entraînement complexe. C'est la raison pour laquelle certains chercheurs tentent de créer des systèmes de représentation graphique plus intuitifs se servant par exemple d'une gamme de couleurs permettant de fournir des informations sur la qualité de l'intonation (Hincks, Edlund 2009).

Cet inventaire succinct des outils informatiques serait incomplet sans une brève mention de systèmes d'évaluation et de correction de la prononciation pleinement autonomes. Leur développement se heurte toujours à deux obstacles majeurs : premièrement, des difficultés d'ordre technique, deuxièmement, des difficultés d'ordre linguistique. En effet, il est nécessaire de répondre à deux questions : quels sont les éléments phonétiques à prendre en considération et à quel modèle les comparer (pour un examen méthodique de ces logiciels voir Luo 2014 : 2-3 ; pour une description détaillée des mécanismes de la reconnaissance vocale et du feedback voir Qian, Meng, Soong 2016). Le développement $\mathrm{d}^{\prime}$ algorithmes de plus en plus puissants et « intelligents" va sans doute rendre possible à l'avenir la création des systèmes permettant un travail pleinement autonome. Cependant, pour l'instant, la situation dans laquelle l'enseignant serait complètement éliminé $\mathrm{du}$ processus de l'apprentissage de la prononciation correcte n'est pas envisageable.

\section{LE DOUBLAGE DANS L'ENSEIGNEMENT DES LANGUES ÉTRANGÈRES}

Le doublage fait partie des tâches dont le principe consiste à manipuler le contenu audiovisuel. De nombreux auteurs se sont penchés sur les bénéfices des activités de ce type. Goodman (2003) considère la création et l'édition des médias comme une des stratégies les plus efficaces dans l'enseignement des langues étrangères. Il le justifie par le fait que les jeunes ressentent un besoin de créer et de partager avec les autres les fruits de leur travail. Kondo (2002), ainsi que Wachob (2010) se concentrent surtout sur le potentiel autonomisant des projets audiovisuels résultant du fait que les élèves décident eux-mêmes de la manière optimale d'accomplir la tâche qui leur est assignée. Pour Górska (2009), l'avantage majeur des projets audiovisuels réside dans l'augmentation de la confiance envers l'enseignant ainsi que l'approfondissement des liens entre les élèves, ce qui rend possible une coopération efficace au sein de la communauté des apprenants. 
Les activités ayant pour objectif la manipulation de la bande sonore d'un enregistrement préexistant peuvent prendre trois formes différentes. Premièrement, les apprenants peuvent inventer de toutes pièces un dialogue et le mettre ensuite dans la bouche des personnages qui apparaissent à l'écran (Kotuła 2014); deuxièmement, ils peuvent préparer une traduction de la bande sonore originale du film et la substituer à l'original (Danan 2010) ; troisièmement, ils peuvent imiter la bande sonore de l'original, ce qui constitue une façon intéressante de travailler efficacement sur le perfectionnement de la prononciation. C'est justement sur cette dernière démarche que nous allons nous focaliser dans le présent article.

Dans le très large éventail d'activités audiovisuelles, le doublage occupe une place peu privilégiée. La littérature dédiée spécifiquement à la réalisation des projets de ce genre dans le cadre de l'enseignement/apprentissage des langues étrangères est plutôt pauvre. Pourtant, comme le remarque avec raison Burston,

phonetic accuracy, stress placement, intonation, rhythm, timing as well as paralinguistic voice features (surprise, sadness, joy, impatience, frustration, etc.) need to be well practiced. In this regard, those dubbing from an existing soundtrack have the advantage of a readily accessible model to follow. (Burston $2005: 84$ )

Le doublage semble donc être bien adapté au travail sur l'intonation et sur l'expression des émotions, car un enregistrement original constitue un excellent modèle à suivre pour les apprenants. Dans la même veine, Chiu (2012) insiste sur la possibilité, offerte par le doublage, d'imiter les locuteurs natifs évoluant dans un contexte réel et palpable. Cette possibilité entre en contraste avec les activités de classe ordinaires dans lesquels les élèves sont priés de répéter des phrases et des fragments de phrase sans ancrage contextuel. Le fait que la bande sonore préparée par les élèves doit correspondre à l'image - dont le contenu est souvent expressif (gestes, expressions faciales, etc.) - exige des participants de s'impliquer réellement dans leur travail pour produire des résultats convaincants.

Un élément important à prendre en considération dans ce contexte est la durée et le rythme du film. Comme le remarque Chiu, " given the time constraints of a rolling film, speaking must occur within a limited period of time » (Chiu 2012 : 26). L'ajustement nécessaire du débit de la parole au contexte visuel rend la tâche particulièrement difficile et exige souvent un long entraînement. Une fois le travail de préparation terminé, le doublage peut être présenté de deux manières distinctes : soit sous forme d'une déclamation ou de la lecture d'un texte en classe alors que la vidéo est projetée sur un écran, ce qui permet aux camarades de classe de suivre la présentation (Chiu 2012), soit après la préparation préalable de la piste sonore et son 
intégration avec la piste image à l'aide d'un logiciel spécialisé. Wakefield fait observer avec raison que la préférence pour l'un ou l'autre mode de présentation peut dépendre du niveau de langue tel que le perçoit l'élève :

For confident learners who want to push themselves, this [la nécessité d'ajuster le débit de la parole au rythme du film lors de lecture synchronisée] could be seen as an advantage because it forces them to perform at a native-speaker pace any failings in accuracy can be worked on with additional practice. However, less confident and less advanced learners benefit from being able to pause, which can only be done with dubbing if they are allowed to pause the video, and doing so will not be as natural as pausing while acting. (Wakefield $2014: 164$ )

Parmi les principaux avantages potentiels du doublage sont énumérés le plus souvent l'augmentation de la motivation ainsi que la réduction du niveau de stress. Burston observe que « even otherwise timid students can take great pleasure seeing themselves perform at their very best, really sounding like authentic native speakers" (Burston 2005: 89). Dans la même veine, Gilet déclare que " l'aspect ludique de l'activité, lié au jeu d'imitation et au processus d'identification, favorise la spontanéité et permet aux élèves de surmonter certains blocages comme la peur du ridicule » (Gilet 2012 : 151). L'aspect ludique mentionné par Gilet est également évoqué par Danan qui, en analysant les réponses fournies par les participants de la recherche qu'il a menée, souligne que "most participants perceived dubbing as a refreshing and entertaining group activity that allowed them to "have fun with the target language" without having to be "quizzed or drilled" » (Danan $2010: 452$ ).

Tous ces avantages ne signifient pas que les projets de doublage doivent réussir à tous les coups. Le succès de l'entreprise dépend en grande partie de la capacité à choisir une vidéo appropriée. Wakefield souligne que «the selection of a video should take into consideration the learners' language level, their language-learning needs and their interests » (Wakefield 2014 : 161). La durée est aussi un facteur crucial ; comme le souligne Danan, "most excerpts should (...) be restricted in length to avoid an overly complex task" (Danan 2010 : 453). Quant aux difficultés potentielles, Wakefield remarque que « some may consider the process of dubbing preparation to be difficult and time-consuming. It requires learners or teachers to have and know how to use video-editing software» (Wakefield 2014: 164). L'enseignant doit naturellement prendre en considération ces aspects qui ont une incidence déterminante sur le déroulement de la tâche.

La plupart des études que nous venons de citer présentent des réflexions d'ordre général sur les avantages potentiels du doublage. À notre connaissance, trois auteurs seulement ont tenté de mener une étude empirique et d'en présenter les résultats d'une manière systématique. Dans sa recherche de 2012, 
Chiu a recueilli les opinions de 83 étudiants d'une université taïwanaise sur leur manière préférée de travailler sur la prononciation. La tâche qu'ont dû réaliser les participants consistait à effectuer le doublage synchronisé d'un fragment de film devant la classe réunie. Comme l'a constaté l'auteur, « analysis of the questionnaire strongly suggests that students consider film dubbing tasks to be an effective method of improving pronunciation » (Chiu 2012:25). La deuxième étude, menée par Huang et Hung (2015), s'est également fondée sur un questionnaire recueillant les opinions des participants. Un groupe de 76 étudiants taïwanais ont dû réaliser une tâche consistant à remplacer la piste sonore d'un fragment de film avec leur propre enregistrement. En analysant les réponses fournies par les élèves, les auteurs sont parvenus à cette conclusion : "students perceived the video-dubbing task as an educational and entertaining task that could function to enhance linguistic gains, decrease language anxiety, promote cultural understanding, strengthen communication confidence, increase learning motivation, reinforce learner autonomy, and foster an enhanced sense of learning community » (Huang, Hung 2015: 287-288). Quant à la troisième étude, de Golshan et Nosratzadeh (2015), elle a été menée sur un échantillon de 60 étudiants iraniens. Les auteurs ont tenté de mesurer l'impact de trois stratégies distinctes (enregistrement de la voix, shadowing et doublage) sur l'amélioration de la qualité de la prononciation des participants. Les deux dernières techniques se sont avérées les plus efficaces. Cette observation a poussé les auteurs à constater que «by having learners apply these techniques in the listening and speaking courses of universities, instructors could get better results pronunciation wise ».

À notre connaissance, aucune étude publiée jusqu'ici ne se concentre sur les variables susceptibles d'influencer la perception des projets de doublage par les participants. Notre étude est une tentative, certes modeste, de combler cette lacune importante.

\section{4. ÉTUDE EMPIRIQUE}

\subsection{Participants et contexte de la recherche}

Les participants à la recherche étaient 24 étudiants de première année en linguistique appliquée à l'Université Marie Curie-Skłodowska à Lublin, en Pologne. Ils étaient âgés de 19 à 23 ans (moyenne 19,80; écart type 0,86). Leur niveau de maîtrise de langue française était à peu près comparable ( B1), même si les parcours qu'ils ont suivis étaient différents : ainsi, ils ont accumulé entre trois et douze années d'étude de français (moyenne 4,33; écart type 2,26). Le groupe était composé majoritairement de filles (22 contre 2 garçons seulement). 
Tous les étudiants ont suivi un cours de phonétique pratique du français au cours de l'année 2015/2016. Les dernières semaines du semestre d'été ont été principalement consacrées au travail sur les traits suprasegmentaux tels que l'intonation et le développement de la capacité à reconnaître et exprimer les émotions particulières. La plupart des exercices ont été introduits en se fondant sur le livre Phonétique progressive du français niveau avancé (Charliac, Motron 2006) dont la troisième partie est spécifiquement consacrée au problème de l'expression des émotions.

\subsection{Description de la tâche proposée}

La tâche qu'ont dû réaliser les participants consistait à substituer la piste sonore originale de deux fragments d'une série télévisée française (Scènes de ménages), produite et diffusée par la chaîne M6. Cette série a été choisie pour trois raisons distinctes. Premièrement, tous les épisodes sont composés d'une suite de courtes scènes, dont chacune constitue une petite histoire complète. Le fait qu'il s'agisse d'unités de sens autonomes facilite leur utilisation dans les projets de doublage; en effet, le travail sur un fragment d'une œuvre plus longue - souvent difficile à situer dans un contexte précis - peut s'avérer problématique. Deuxièmement, la durée de la plupart des scènes ne dépasse pas une minute. $C^{\prime}$ est un paramètre crucial vu la quantité de travail que demande la réalisation des projets de doublage. Troisièmement, la thématique de la série ainsi que la nature des scènes les prédisposent à être utilisés dans le contexte didactique: il s'agit de situations impliquant deux ou plusieurs personnes où sont juxtaposées dans un laps de temps très court des réactions très variées telles que la joie, la colère, la surprise, l'étonnement, etc. Le caractère très dynamique des scènes permet aux élèves d'imiter toute une palette d'émotions.

$\mathrm{Vu}$ que les deux scènes choisies présentaient des dialogues entre les couples, les participants ont dû réaliser leur tâche en paires. Les élèves étaient libres de choisir leur partenaire. Le contenu des scènes, qui ont été sélectionnées par l'enseignant, a été analysé pendant un des cours. La transcription des dialogues a été effectuée et les arrangements intonatifs ont été identifiés. Une fois ce travail accompli, les deux enregistrements ont été " mis en nuage » pour pouvoir être facilement accessibles à tous les participants. Comme les élèves avaient déjà une expérience préalable dans la préparation des enregistrements sonores (ils avaient été enregistrés en classe et avaient préparé des enregistrements sonores à la maison), ils n'avaient pas besoin d'une formation particulière dans ce domaine. Par contre, la tâche consistant à substituer la bande sonore d'un enregistrement audiovisuel était 
pour eux une nouveauté. C'est pour cette raison que deux tutoriels en français leur ont été fournis: le premier présentant les fonctionnalités de base du logiciel Audacity, permettant de modifier les enregistrements audio (p. ex. couper les fragments inutiles, manipuler le volume sonore, etc.), le deuxième se focalisant sur Microsoft Movie Maker, permettant d'assembler le produit final. La durée du projet a été fixée à cinq semaines.

\subsection{Description du questionnaire}

Une fois le travail terminé, les étudiants ont été priés de remplir un questionnaire composé de 28 items. Dans la première partie de l'enquête, les participants ont dû fournir quelques informations essentielles, telles que sexe ou la durée de l'apprentissage du français. Ensuite, ils ont été priés de prendre position par rapport à une série de 24 assertions se référant à leurs opinions sur le projet proposé ainsi qu'à leur capacité de prononcer correctement les sons de la langue française et l'importance de la prononciation correcte dans la communication orale. Pour chacun des items, le choix de réponse s'effectuait sur une échelle de type Likert de cinq points qui prévoyait une modalité de réponse de 1 (pas du tout d'accord) à 5 (tout à fait). Ces assertions sont reproduites dans leur intégralité dans le Tableau 2. Pour toutes les analyses statistiques, le logiciel Statistica version 8 a été utilisé.

\subsection{Questions de recherche}

Les principales questions de recherche auxquelles cette étude tentait de répondre étaient les suivantes :

1) Comment les projets de doublage sont-ils perçus par les apprenants de la langue française ? Les considèrent-ils comme une manière intéressante et efficace de travailler sur la prononciation française?

2) Quelles sont les variables qui peuvent avoir un impact sur les opinions exprimées par les participants ? Existe-t-il une relation entre certaines convictions des étudiants relatives à l'apprentissage des langues étrangères et leur avis sur le projet proposé ?

\subsection{Présentation des résultats}

En premier lieu, nous allons nous pencher sur les réponses fournies par les participants aux 8 items du questionnaire directement liées à la perception du projet. L'ensemble des données est présenté dans le Tableau 1 ci-dessous. 
Tableau 1. Moyennes, valeurs minimales et maximales et écart type pour chacun des items se référant à l'appréciation du projet par les participants

\begin{tabular}{|l|c|c|c|c|c|}
\hline \multicolumn{1}{|c|}{ Variable } & $\mathrm{N}$ & Moyenne & Min & Max & $\begin{array}{c}\text { Écart } \\
\text { type }\end{array}$ \\
\hline La réalisation de ce projet a été difficile & 24 & 3,66 & 2,00 & 5,00 & 1,20 \\
\hline La réalisation de ce projet a été chronophage & 24 & 4,41 & 2,00 & 5,00 & 1,01 \\
\hline $\begin{array}{l}\text { Au cours de la réalisation du projet, nous } \\
\text { avons dû faire face à de nombreux problèmes } \\
\text { techniques }\end{array}$ & 24 & 3,29 & 1,00 & 5,00 & 1,68 \\
\hline $\begin{array}{l}\text { Je suis satisfait des résultats du travail de mon } \\
\text { groupe }\end{array}$ & 24 & 4,16 & 2,00 & 5,00 & 0,86 \\
\hline $\begin{array}{l}\text { Je suis satisfait de ma contribution personnelle } \\
\text { au projet }\end{array}$ & 24 & 4,37 & 2,00 & 5,00 & 0,82 \\
\hline $\begin{array}{l}\text { La coopération au sein de notre groupe était } \\
\text { satisfaisante }\end{array}$ & 24 & 4,91 & 4,00 & 5,00 & 0,28 \\
\hline $\begin{array}{l}\text { La participation à ce projet m'a permis } \\
\text { d'améliorer mon intonation du français }\end{array}$ & 24 & 3,83 & 2,00 & 5,00 & 0,91 \\
\hline $\begin{array}{l}\text { La participation à ce projet m'a permis } \\
\text { d'améliorer ma prononciation du français }\end{array}$ & 24 & 3,95 & 2,00 & 5,00 & 1,04 \\
\hline
\end{tabular}

Comme nous pouvons le constater, l'avis des étudiants sur le degré de difficulté générale (moyenne 3,66), ainsi que sur le degré de difficulté technique (moyenne 3,29) était plutôt neutre, indiquant que les participants n'ont pas rencontré d'obstacles sérieux au cours de la réalisation du doublage. Pourtant, les élèves ont affirmé que l'exécution de la tâche leur a pris beaucoup de temps (moyenne 4,41). Ceci n'est guère surprenant: les deux phases essentielles de la réalisation du projet, à savoir l'entraînement ainsi que la production et l'édition de l'enregistrement sonore, sont chronophages. Quant au niveau de satisfaction exprimé par les participants de la recherche, il est assez élevé : les étudiants étaient contents aussi bien de la qualité du produit final (moyenne 4,16) que de la manière dont ils ont contribué à la réalisation du projet (moyenne 4,37 ). Seule une personne a avoué ne pas être entièrement contente des résultats du travail de son groupe ainsi que de sa contribution personnelle au projet. Le plus grand niveau de satisfaction a été affiché à l'égard de la qualité de la coopération au sein du groupe (moyenne 4,91). Ceci a peut-être résulté du fait que les participants ont pu choisir librement leur partenaire, ce qui a probablement contribué à leur confort. Les deux derniers items, se référant directement à l'impact perçu du doublage sur le perfectionnement de la prononciation et de l'intonation vont être analysés plus en détail sur le graphique 1 . 


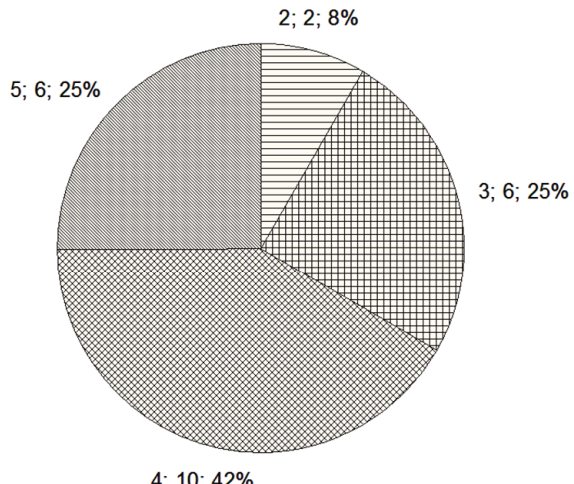

$4 ; 10 ; 42 \%$

Intonation

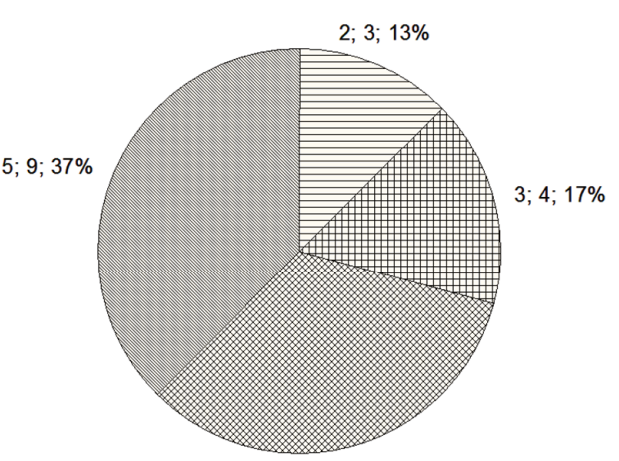

$4 ; 8 ; 33 \%$

Prononciation

Graphique 1. Diagrammes circulaires montrant la répartition des réponses fournies par les participants à la question « La participation à ce projet $\mathrm{m}$ 'a permis d'améliorer mon intonation $\mathrm{du}$ français » (à gauche) et « La participation à ce projet m'a permis d'améliorer ma prononciation du français » (à droite). Le premier chiffre correspond à la réponse choisie par le participant, le deuxième indique le nombre de participants qui ont fourni cette réponse, le troisième montre le pourcentage correspondant

Comme nous pouvons le constater, la plupart des participants ont exprimé un avis positif ou très positif quant à l'impact potentiel du doublage sur l'amélioration de leur capacité de prononcer correctement les sons du français $(70 \%)$ et sur leur intonation (67\%). Aucun des étudiants n'a exprimé $\mathrm{d}$ 'avis entièrement négatif sur le projet. Cependant, quelques participants ont exprimé un avis plutôt négatif ( 2 dans le cas de l'intonation et 3 dans le cas de la prononciation) ou neutre (6 dans le cas de l'intonation et 4 dans le cas de la prononciation). En général, il faut conclure que la réception du projet par les étudiants a été plutôt positive.

L'étape suivante de l'analyse a consisté en une étude corrélationnelle des variables. Nous avons pris comme point de repère principal les deux items se référant directement à la perception du projet par les étudiants, à savoir les variables « La participation à ce projet m'a permis d'améliorer mon intonation du français » et «La participation à ce projet m'a permis d'améliorer ma prononciation du français ». Comme les variables que nous avons obtenues n'étaient pas distribuées selon la loi normale, le test non-paramétrique de corrélation de Spearman a été utilisé. L'ensemble des données est présenté dans le Tableau 2 ci-dessous.

Les deux premières variables, "sexe " et "âge ", ne feront pas l'objet d'une analyse particulière. Ceci est justifié par le fait que seuls deux participants de sexe masculin ont pris part à la recherche. De même, comme notre 
Tableau 2. Valeur du coefficient de Spearman pour les corrélations choisies. Sont marquées avec un astérisque les corrélations dont le coefficient $\mathrm{p}$ était $<0,05$

\begin{tabular}{|c|c|c|}
\hline Variable & $\begin{array}{l}\text { La participation à ce } \\
\text { projet m'a permis } \\
\text { d'améliorer mon into- } \\
\text { nation du français }\end{array}$ & $\begin{array}{l}\text { La participation à ce } \\
\text { projet m'a permis } \\
\text { d'améliorer ma pro- } \\
\text { nonciation du français }\end{array}$ \\
\hline Sexe & $-0,41^{*}$ & $-0,34$ \\
\hline Âge & 0,31 & 0,04 \\
\hline Depuis combien d'années apprenez-vous le français? & $-0,24$ & $-0,39$ \\
\hline $\begin{array}{l}\text { Combien de temps avez-vous passé dans un pays franco- } \\
\text { phone? }\end{array}$ & 0,21 & 0,17 \\
\hline Je parle et écris le français couramment & 0,31 & $-0,04$ \\
\hline Ma prononciation française est très bonne & $-0,18$ & $-0,15$ \\
\hline La prononciation française est très difficile & 0,17 & $0,55^{*}$ \\
\hline J'aime participer à différents projets & 0,20 & $-0,03$ \\
\hline $\begin{array}{l}\text { Je crois pouvoir maîtriser couramment la prononciation } \\
\text { française dans l'avenir }\end{array}$ & 0,38 & 0,24 \\
\hline J'aime travailler en groupe & 0,23 & 0,34 \\
\hline $\begin{array}{l}\text { J'essaie d'avoir le plus de contact possible avec la langue } \\
\text { française en dehors de l'école }\end{array}$ & 0,18 & 0,06 \\
\hline $\begin{array}{l}\text { Une/La prononciation correcte de la langue étrangère est } \\
\text { très importante }\end{array}$ & 0,22 & $-0,09$ \\
\hline $\begin{array}{l}\text { L'enseignement des langues étrangères à l'aide de nouvel- } \\
\text { les technologies peut être bien plus efficace que } \\
\text { l'enseignement traditionnel }\end{array}$ & $0,41^{*}$ & 0,07 \\
\hline $\begin{array}{l}\text { Je crois savoir me servir d'un ordinateur d'une manière } \\
\text { efficace }\end{array}$ & 0,26 & $-0,01$ \\
\hline Je suis satisfait des résultats du travail de mon groupe & $0,44^{*}$ & $-0,21$ \\
\hline Je suis satisfait de ma contribution personnelle au projet & 0,25 & $-0,19$ \\
\hline $\begin{array}{l}\text { La prononciation française de mon partenaire est très } \\
\text { bonne }\end{array}$ & 0,13 & $-0,35$ \\
\hline La réalisation du doublage a été très chronophage & $-0,19$ & 0,03 \\
\hline $\begin{array}{l}\text { Je ne me sens pas à l'aise lorsque j'écoute le son de ma } \\
\text { voix sur les enregistrements }\end{array}$ & 0,18 & $-0,38$ \\
\hline $\begin{array}{l}\text { J'ai l'impression que je n'apprendrai jamais à prononcer } \\
\text { correctement certains sons de la langue française }\end{array}$ & $-0,44^{*}$ & $-0,05$ \\
\hline L'intonation française me pose de sérieux problèmes & $-0,31$ & $-0,03$ \\
\hline La réalisation du doublage a été difficile & $-0,33$ & $-0,04$ \\
\hline $\begin{array}{l}\text { Au cours de la réalisation du doublage, nous avons ren- } \\
\text { contré de sérieux problèmes techniques }\end{array}$ & $-0,14$ & 0,10 \\
\hline $\begin{array}{l}\text { La participation à ce projet } \mathrm{m}^{\prime} \text { a permis d'améliorer mon } \\
\text { intonation du français }\end{array}$ & 1,00 & $0,44^{*}$ \\
\hline
\end{tabular}




\begin{tabular}{|c|c|c|}
\hline Variable & $\begin{array}{l}\text { La participation à ce } \\
\text { projet m'a permis } \\
\text { d'améliorer mon into- } \\
\text { nation du français }\end{array}$ & $\begin{array}{l}\text { La participation à ce } \\
\text { projet m'a permis } \\
\text { d'améliorer ma pro- } \\
\text { nonciation du français }\end{array}$ \\
\hline La coopération au sein du groupe s'est très bien déroulée & 0,16 & $-0,07$ \\
\hline $\begin{array}{l}\text { J'ai l'impression d'avoir fait d'importants progrès de } \\
\text { prononciation au cours de cette année }\end{array}$ & $0,46^{*}$ & 0,26 \\
\hline $\begin{array}{l}\text { La participation à ce projet m'a permis d'améliorer ma } \\
\text { prononciation du français }\end{array}$ & $0,44^{*}$ & 1,00 \\
\hline $\begin{array}{l}\text { Je crois qu'un travail régulier sur la prononciation pen- } \\
\text { dant les cours apporte de meilleurs résultats que la réali- } \\
\text { sation de projets de ce type }\end{array}$ & $-0,39$ & $-0,30$ \\
\hline
\end{tabular}

échantillon était homogène en ce qui concerne l'âge (cf. section 4.1.), nous avons écarté cette variable de l'étude.

Comme nous pouvons l'observer, la plupart des corrélations (48;86\%) se sont avérées trop faibles pour atteindre la signification statistique. Toutes les corrélations statistiquement significatives $(8 ; 14 \%)$ sont de force modérée.

La première des variables se rapportant directement aux avantages perçus du projet proposé, "la participation à ce projet m'a permis d'améliorer mon intonation du français », corrèle d'une manière significative avec cinq autres variables. La première de ces corrélations, avec la variable « l'enseignement des langues étrangères assisté de nouvelles technologies peut être bien plus efficace que l'enseignement traditionnel », est positive $\left(r_{s}=0,41\right)$. Elle indique que plus les sujets étaient convaincus de l'utilité des nouvelles technologies dans l'apprentissage de langues, plus le projet proposé leur a semblé avantageux dans le contexte du travail sur l'intonation. D'autre part, il en résulte que les individus attachés à des méthodes traditionnelles de travail de la prononciation ont été moins enclins à participer à des projets de doublage. La deuxième corrélation, avec la variable « je suis satisfait des résultats du travail de mon groupe », est aussi positive $\left(r_{s}=0,44\right)$. Elle témoigne de l'existence d'une relation proportionnelle entre le niveau de satisfaction relative à la qualité du projet final et la conviction des étudiants que la participation à ce projet leur a permis d'améliorer leur intonation. La troisième corrélation statistiquement significative, avec la variable « j'ai l'impression que je n'apprendrai jamais à prononcer correctement certains sons de la langue française", est négative $\left(r_{s}=-0,44\right)$. Elle montre que la conviction des participants relative à leur difficulté à maîtriser la prononciation correcte des sons de la langue française est inversement proportionnelle à leur opinion sur l'impact du projet sur leur maîtrise de l'intonation. Ainsi, plus l'absence d'auto-confiance des sujets était marquée, moins ils étaient 
persuadés de l'intérêt de la tâche proposée. La quatrième corrélation s'observe dans le cas de la variable « j'ai l'impression d'avoir fait d'importants progrès de prononciation au cours de cette année » $\left(\mathrm{r}_{\mathrm{s}}=0,46\right)$. Elle indique l'existence d'une relation positive entre le sentiment d'avoir accompli d'importants progrès au cours de l'année universitaire 2015/2016 et la conviction concernant le caractère bénéfique du projet. La dernière corrélation, avec la variable « la participation à ce projet m'a permis d'améliorer ma prononciation du français ", est aussi positive $\left(r_{s}=0,44\right)$. La force modérée de cette corrélation est intéressante : il en résulte que les personnes participant à la recherche ont établi une différence nette entre l'impact du projet sur l'amélioration de la prononciation et l'impact du projet sur l'amélioration de l'intonation.

La deuxième variable prise en compte dans notre analyse, « la participation à ce projet m'a permis d'améliorer ma prononciation du français ", corrèle d'une manière significative avec deux variables seulement. La première corrélation, avec la variable « la prononciation française est très difficile », est positive $\left(r_{s}=0,55\right)$. Elle montre que plus les sujets étaient convaincus de la difficulté de la prononciation française, plus ils ont apprécié l'impact du projet sur leur capacité à prononcer correctement les sons de la langue française. La deuxième corrélation, avec la variable «la participation à ce projet m'a permis d'améliorer mon intonation du français ", a déjà été commentée dans le paragraphe précédent.

L'analyse de certaines corrélations qui se sont avérées non-significatives apporte aussi quelques renseignements éclairants. Premièrement, il est important de souligner que ni le temps d'étude de la langue française ni la quantité de temps passé dans un pays francophone n'ont eu d'influence sur les opinions exprimées par les participants de la recherche. De la même façon, les variables telles que «je parle et écris le français couramment» ou «ma prononciation française est très bonne » n'ont pas corrélé de manière significative avec l'impact perçu du projet sur l'amélioration de la qualité de la prononciation et de l'intonation. Cela prouve que les projets de doublage peuvent être considérés comme intéressants par les élèves à des niveaux de langue variés. Ensuite, aucune corrélation statistiquement significative n'a été décelée dans le cas des variables "j'aime participer à différents projets » et "j'aime travailler en groupes». Cela prouve que les tâches de ce type peuvent aussi être perçues comme intéressantes par des personnes n'appréciant pas particulièrement la coopération au sein d'une communauté d'apprenants. Finalement, aucune relation statistiquement significative n'a été trouvée dans le cas de la variable « je crois savoir me servir d'un ordinateur d'une manière efficace ", ce qui indique que la perception du projet ne dépendait pas des compétences informatiques des sujets. 


\section{CONCLUSIONS}

Les projets de doublage présentent de nombreux avantages potentiels. Ils permettent aux apprenants de travailler sur des ressources audiovisuelles ancrées dans un contexte langagier authentique. Ils leur offrent également une opportunité de créer quelque chose de concret et de partager les fruits de leur travail avec les autres. Finalement, surtout dans le contexte de travail sur la prononciation, les tâches de ce type ont l'avantage indéniable de briser la routine de classe en proposant aux élèves des activités bien plus stimulantes que des exercices d'écoute ou d'imitation répétitifs et ennuyeux.

Les résultats des recherches que nous avons citées dans la section 3 ainsi que ceux de l'étude que nous avons menée semblent indiquer que la majorité des apprenants considèrent le doublage comme une façon intéressante et efficace de travailler sur la prononciation. Le succès des projets de ce type dépend inévitablement de nombreuses variables, telles que les caractéristiques du groupe, les objectifs visés, la nature du document audiovisuel choisi, etc. Le rôle de l'enseignant paraît dans ce contexte particulièrement important. Il lui incombe de veiller au bon déroulement de la tâche à chacune de ses étapes, depuis la sélection d'un film adapté aux besoins des apprenants jusqu'à l'évaluation de l'effet final. Le soutien et l'assistance technique offerts aux élèves par le professeur sont également essentiels. Toutes ces exigences ne devraient cependant pas décourager les enseignants d'introduire des innovations dans le processus didactique, car les résultats peuvent se révéler très positifs.

\section{RÉFÉRENCES}

Baran-Łucarz, M. (2006). Prosto w oczy - fonetyka jako 'Michałek' na studiach filologicznych? In: W. Sobkowiak / E. Waniek-Klimczak (eds.). Dydaktyka fonetyki języka obcego w Polsce. Konin: Wydawnictwo PWSZ, 7-17.

Burston, J. (2005). Video dubbing projects in the foreign language curriculum. CALICO Journal, $23(1), 79-92$.

Charliac, L. / Motron, A.C. (2006). Phonétique progressive du français. Niveau avancé. Paris : CLE International.

Chiu, Y. (2012). Can film dubbing projects facilitate EFL learners' acquisition of English pronunciation? British Journal of Educational Technology, 43 (1), pp. 24-27.

Danan, M. (2010). Dubbing projects for the language learner: a framework for integrating audiovisual translation into task-based instruction. Computer Assisted Language Learning, 23 (5), 441-456.

Gilet, G. (2012). Doublage d'un extrait de Volver d'Almodóvar et modification du scénario. Recherche et pratiques pédagogiques en langues de spécialité, 31 (2), 151-159.

Gajewska, E. (2011). Techniki nauczania języka obcego. Tarnów: PWSZ w Tarnowie. 
Golshan, N.S. / Nosratzadeh, H. (2015). The impact of video dubbing, voice recording and shadowing tasks on Iranian EFL learners' command of pronunciation. International Journal of Language and Applied Linguistics, 1, pp. 7-16.

Goodman, S. (2003). Teaching Youth Media: A Critical Guide to Literacy, Video Production, E Social Change. Nowy Jork: Teachers College Press.

Górska, W. (2009). Narzędzia web 2.0 w codziennej pracy nauczyciela języków obcych. Neofilo$\log , 33$, pp. 197-204.

Hermeline, L. (2001). Enseigner la phonétique : oui, mais comment? Le français dans le monde, 318 , pp. 27-28.

Hincks, R. / Edlund, J. (2009). Promoting increased pitch variation in oral presentation with transient visual feedback. Language Learning and Technology, 13 (3), pp. 32-50.

Huang, H.D. / Hung, S.A. (2015). Effects of a video-dubbing task: perspectives of EFL learners. In: J. Colpaert / A. Aerts / M. Oberhofer / M. Gutiérrez-Colón Plana (eds.). Proceedings of the seventeenth International CALL Research Conference, Tarragona, Universitat Rovira $i$ Virgili, 6-8 July 2015. Antwerp: University Press Antwerp. pp. 286-288.

Kondo, I. (2002). Video and learner enthusiasm: Stimulating personal interest as the first step towards autonomy In: A.S. Mackenzie / T. Newfield (eds.). Proceedings of the JALT CUE and TEVAL mini-conferences. Kyoto, Japan, Tokyo: JALT, pp. 83-86.

Kotuła, K. (2014). Les pratiques innovatrices en classe de langues. L'édition et la production des films dans l'enseignement de FLE. Studia Romanica Posnaniensia, 41 (3), pp. 47-61.

Luo, B. (2014). Evaluating a computer-assisted pronunciation training (CAPT) technique for efficient classroom instruction. Computer Assisted Language Learning, 29 (3), pp. 451-476.

Olson, D.J. (2014). Benefits of visual feedback on segmental production in the L2 classroom. Language Learning \& Technology, 18 (3), pp. 173-192.

Parizet, M.L. (2008). Phonétique et Cadre Commun: propositions pour un cours de FLE. Synergies Espagne 1, pp. 113-122.

Qian, X. / Meng H. / Soong, F. (2016). Two-pass framework of mispronunciation detection \& diagnosis for computer-aided pronunciation training. IEEE/ACM Transactions on Audio, Speech, and Language Processing, 24 (6), pp. 1020-1028.

Richards, J.C. / Renandya, W.A. (2002). Methodology in language teaching: An anthology of current practice. Cambridge: Cambridge University Press.

Rojczyk, A. (2011). Wykorzystanie programu do analizy mowy w nauce wymowy języka obcego. Nagłosowy kontrast dźwięczności w języku angielskim. In : M. Pawlak / B. Wolski (eds.). Wykorzystanie nowoczesnych technologii w dydaktyce języków obcych. Konin: Wydawnictwo PWSZ, pp. 65-74.

Wachob, P. (2010). Using videos of students in the classroom to enhance learner autonomy. Teaching English with Technology, 11, pp. 18-28.

Wakefield, J.C. (2014). Dubbing as a method for language practice and learning. In: C. DeCoursey (ed.). Language Arts in Asia 2: English and Chinese through literature, drama and popular culture Newcastle upon Tyne: Cambridge Scholars Publishing, pp. 160-166.

Received: 20.02.2017; revised: 2.04.2017 